\title{
Stability of synchronous states in sparse neuronal networks
}

\author{
Afifurrahman • Ekkehard Ullner(i) - Antonio Politi 1
}

Received: 3 July 2020 / Accepted: 3 August 2020 / Published online: 24 August 2020

(C) The Author(s) 2020

\begin{abstract}
The stability of synchronous states is analyzed in the context of two populations of inhibitory and excitatory neurons, characterized by two different pulse-widths. The problem is reduced to that of determining the eigenvalues of a suitable class of sparse random matrices, randomness being a consequence of the network structure. A detailed analysis, which includes also the study of finite-amplitude perturbations, is performed in the limit of narrow pulses, finding that the overall stability depends crucially on the relative pulsewidth. This has implications for the overall property of the asynchronous (balanced) regime.
\end{abstract}

Keywords Stability analysis · Synchronization · Neuronal network $\cdot$ Sparse network

\section{Introduction}

Networks of oscillators are widely studied in many fields: mechanical engineering [1,2], power grids [3], arrays of Josephson junctions [4], cold atoms [5], neu-

Afifurrahman · E. Ullner $(\varangle) \cdot$ A. Politi

Institute for Pure and Applied Mathematics and

Department of Physics (SUPA), Old Aberdeen, Aberdeen

AB24 3UE, UK

e-mail: e.ullner@abdn.ac.uk

Afifurrahman

e-mail: r01a17@abdn.ac.uk

A.Politi

e-mail: a.politi@abdn.ac.uk ral networks [6], and so on. Such networks can be classified according to the single-unit dynamics, the coupling mechanism, the presence of heterogeneity, and network topology. Since phases are the most sensitive variables to any kind of perturbation [7], most of the attention is devoted to setups composed of phase oscillators [8], i.e., one-dimensional dynamical systems. However, even the study of such relatively simple models is not as straightforward as it might appear. In fact, a wide variety of dynamical regimes can emerge even in mean field models of identical oscillators, ranging from full synchrony to splay states, and including hybrids, such as partial synchronization [9], chimera [10], and cluster states [11]. General theory of synchronization is, therefore, a much investigated field.

In this paper, we focus on synchronous states by referring to a rather popular class of neural networks, but the whole formalism can be easily extended to more general systems so long as the coupling is mediated by the emission of pulses. In neuroscience, the neuron dynamics is often described by a single variable, the membrane potential, which evolves according to a suitable velocity field. The resulting model is equivalent to a phase oscillator, where the variable of the bar system increases linearly in time and the complexity of the evolution rule is encoded in the phase response curve (PRC), which accounts for the mutual coupling [12]. Under the additional approximation of a weak coupling strength, the model can be further simplified and cast into a Kuramoto-Daido form, where the coupling depends on phase differences between pairs of 
oscillators $[13,14]$. Here, however, we stick to pulsecoupled oscillators.

The stability of the synchronized state of pulsecoupled phase oscillators has been first studied in the context of excitatory $\delta$-pulses [15]. Synchronization is induced when two oscillators are sufficiently close and a common excitatory $\delta$-pulse instantaneously sets both to the same value. Later, the stability analysis for excitatory and inhibitory pulses $[16,17]$ has been extended to $\delta$-pulses with continuous PRCs [18]. General formulas are mostly available under severe restrictions, such as identical oscillators, mean field interactions, or $\delta$-like pulses.

The $\delta$-like pulse assumption is particularly limiting, not only because realistic systems are characterized by a finite width, but also because it has been shown that zero-pulsewidth is a singular limit, which does not commute with the thermodynamic limit (infinitely large networks) — at least in the context of splay states [19]. Relaxing the zero-width limit forces to increase the phase-space dimension to account for the dynamics of the fields felt by the different neurons. The most general result we are aware of is a formula derived in Ref. [20] for a single population of identical neurons in the presence of mean-field coupling and the so-called $\alpha$ pulses.

The introduction of sparseness implies a significant increase in the computational complexity because of the randomness of the connections. In this context, the most relevant results are those derived in Ref. [21], where a sparse random network (Erdös-Rényi type) has been investigated in the presence of $\delta$-pulses. The approach is rather complex since the noncommutativity associated with changes in the order of the incoming spikes obliged the authors to introduce a multitude of linear operators to solve the problem.

Here, we extend this kind of stability analysis to finite pulse-widths in two populations of excitatory, respectively, inhibitory, neurons. Our approach can also be considered as an extension to sparse networks of the work in Ref. [20] devoted to mean-field models. This setup is chosen in studies of the so-called balanced state [22], where the asynchronous regime is dominated by strong fluctuations. Typically, the balance depends on both the relative size of the two populations and the relative amplitude of the pulses. In this paper, a careful study of the fully synchronous regime shows that also the relative pulse-width plays a non-trivial role.
Finite-width pulses can obviously have infinitely many different shapes. In this paper, we consider the simplest case of exponential spikes and assume, as usual, that they superpose linearly. In practice, this means that each oscillator (neuron) is characterized by three variables: the phase or, equivalently, the membrane potential and two variables describing the incoming excitatory and inhibitory fields, respectively. At variance with Ref. [20], instead of transforming the model into a mapping (from one to the next spike emission), here we preserve the time continuity, as this approach allows for a more homogeneous treatment of the oscillators maintaining the full $3 N$ dimensional structure of the phase-space (where $N$ is the number of oscillators).

Furthermore, in agreement with previous publications [23-25] we assume that each neuron receives exactly the same number of excitatory and inhibitory synaptic connections. In fact, in spite of the random connectivity, in the fully synchronous regime, all neurons are characterized by exactly the same variables. A crucial difference with respect to the mean-field model is that the degeneracy of the Lyapunov spectrum is lifted and the stability assessment amounts to determining the eigenvalues of a suitable (sparse) random matrix.

More precisely, in Sect. 2 we define the model, including the specific phase response curve used to perform numerical tests. The overall linear stability analysis is discussed in Sect. 3, first with reference to the general case and then specifically referring to short (but finite) pulses. In the same section, we also determine the conditional Lyapunov exponent $\lambda_{c}$, (i.e., the exponent of a single neuron subject to a given periodic forcing): at variance with the mean-field model, $\lambda_{c}$ differs from the maximum exponent of the whole network, meaning that the connectivity plays a crucial role. In Sect. 4, we implement the formulas determined in the previous section to discuss the qualitative changes observed by varying the relative pulse-width. Finally, Sect. 5 is devoted to a summary of the main results and an outline of the open problems.

\section{Model}

The object of study is a network of $N$ phase-oscillators (also referred to as neurons), the first $N_{e}$ being excitatory, the last $N_{i}$ inhibitory (obviously, $N_{e}+N_{i}=N$ ). 
Each neuron is characterized by the phase-like variable $\Phi^{j} \leq 1$ (formally equivalent to the membrane potential), while the (directed) synaptic connections are represented by the connectivity matrix $\mathbf{G}$ with the entries

$G_{j, k}= \begin{cases}1, & \text { if } k \rightarrow j \text { active } \\ 0, & \text { otherwise }\end{cases}$

where $\sum_{k \geq 1}^{N_{e}} G_{j, k}=K_{e}$ and $\sum_{k>N_{e}}^{N} G_{j, k}=K_{i}$, meaning that each neuron $j$ is characterized by the same number of incoming excitatory and inhibitory connections, as customary assumed in the literature [23] ( $K=K_{e}+K_{i}$, finally, represents the connectivity altogether).

The evolution of the phase of both excitatory and inhibitory neurons is ruled by the same equation,

$\dot{\Phi}^{j}=1+J \Gamma\left(\Phi^{j}\right)\left(E^{j}-I^{j}\right)$,

where $\Gamma(\Phi)$ represents the phase-response curve (PRC), $J$ the coupling strength and $E^{j}\left(I^{j}\right)$ the excitatory (inhibitory) field generated by the incoming connections. Scaling properties with the network size $N$ affect the coupling $J$. In the case of sparse networks with fixed connectivity (the case herein discussed), $K$ as well as $J$ are simply independent of $N$. Whenever $\Phi^{j}$ reaches the threshold $\Phi_{t h}=1$, the phase is reset to $\Phi_{r}=0$ and enters a refractory period $t_{r}$ during which it stands still and is insensitive to the action of the excitatory $\left(E^{j}\right)$ and inhibitory $\left(I^{j}\right)$ field. At the same time, the fields of the receiving neurons are activated. If the neuron $k$, emitting a spike at time $t_{n}^{k}$, is excitatory $\left(k \leq N_{e}\right)$, then the excitatory field $E^{j}$ of any receiving neuron $j$ is activated (and similarly for the inhibitory field $I^{j}$ ).

The fields in Eq. (1) evolve according to the differential equations

$$
\begin{aligned}
\dot{E}^{j} & =-\alpha\left(E^{j}-\sum_{n} G_{j, k} P_{k, k} \delta\left(t-t_{n}^{k}\right)\right) \\
\dot{I}^{j} & =-\beta\left(I^{j}-g \sum_{n} G_{j, k}\left(\delta_{k, k}-P_{k, k}\right) \delta\left(t-t_{n}^{k}\right)\right),
\end{aligned}
$$

where $\alpha(\beta)$ denotes the inverse pulse-width of the excitatory (inhibitory) spikes. The coefficient $g$ accounts for the relative amplitude of inhibitory spikes compared to excitatory ones. $P_{k, m}$ represents the elements of a projector operator $\mathbf{P}$, separating excitatory from inhibitory neurons: $P_{k, m}=0$ except when $k=m \leq$ $N_{e}$, in which case $P_{k, k}=1$.

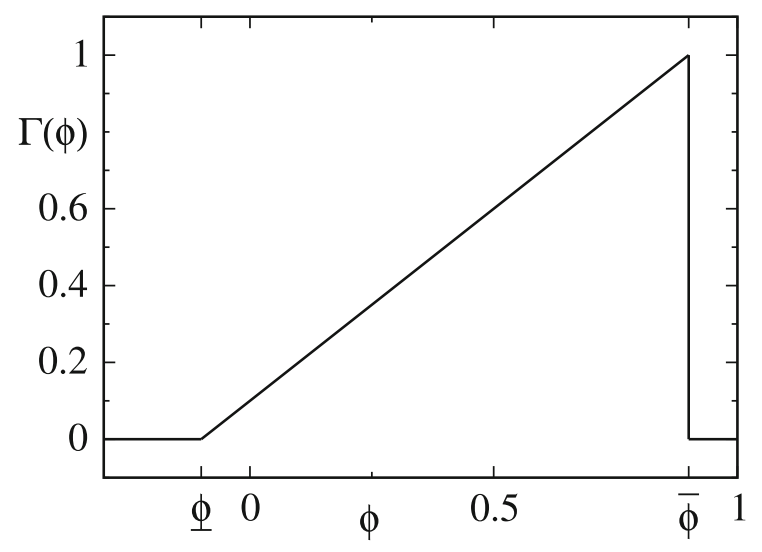

Fig. 1 Example of the phase response curve (PRC) used in Sect. 4 with $\underline{\Phi}=-0.1, \bar{\Phi}=0.9$ in combination with $\Phi_{r}=0$ and $\Phi_{t h}=1$

In order to be more specific, we introduce the PRC used later on as a testbed for the formalism developed in the next section. We have chosen to work with the following piecewise linear PRC,

$\Gamma\left(\Phi^{j}\right)= \begin{cases}\left(\Phi^{j}-\underline{\Phi}\right) & \text { if } \underline{\Phi}<\Phi^{j}<\bar{\Phi} \\ 0 & \text { otherwise }\end{cases}$

where $\underline{\Phi}<0$, and $0<\bar{\Phi}<1$ characterize the PRC. The resulting shape is plotted in Fig. 1 for $\underline{\Phi}=-0.1$ and $\bar{\Phi}=0.9^{1}$.

As anticipated in the introduction, we are interested in assessing the stability of the fully synchronous dynamics of period $T$ as a function of the relative pulsewidth, where $T$ is the interspike interval. The solution is obtained by integrating the equation,

$\left\{\begin{array}{l}\dot{\Phi}=1+J \Gamma(\Phi)(E-I) \\ E(t)=E_{\circ} \mathrm{e}^{-\alpha t} \\ I(t)=I_{\circ} \mathrm{e}^{-\beta t}\end{array}\right.$

where

$E_{\circ}=\frac{K_{e} \alpha}{1-\mathrm{e}^{-\alpha T}}, \quad I_{\circ}=\frac{g K_{i} \beta}{1-\mathrm{e}^{-\beta T}}$

are the magnitudes of the fields immediately after the synchronous spike emission. The constants $E_{\circ}$ and $I_{\circ}$ result self-consistently from the remaining field at the

1 The peculiarity of a vanishing PRC for $\bar{\Phi}<\Phi \leq 1$ is introduced to match the PRC recently introduced in [26] to mimic delay-coupled leaky integrate-and-fire neuron. However, it does not affect the generality of the formalism, as it can be easily "removed" by assuming $\bar{\Phi}=1$. 


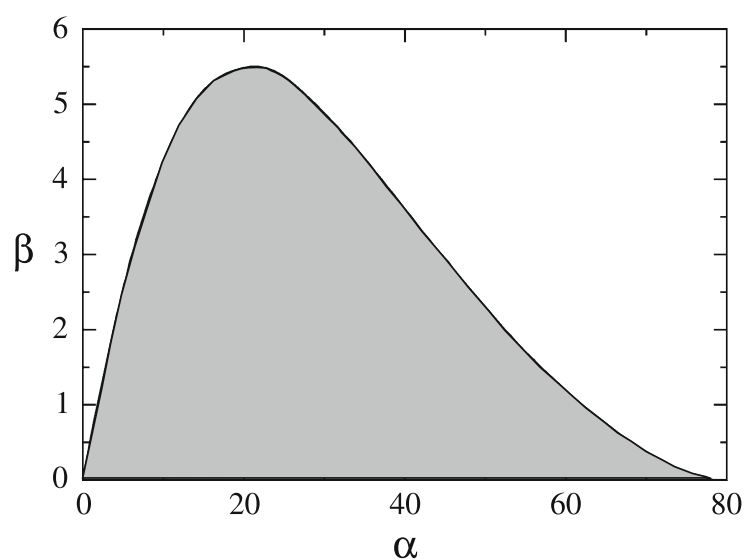

Fig. 2 Phase diagram of synchronous regimes. Period-1 solutions exist above the curve in the white area. Crossing the curve, in the grey area, period-2 solutions first emerge followed (further down) by longer period regimes

end of the period $T$ plus the contribution from the spike emission.

In the present paper, we focus on the stability of the synchronous period-1 solution (i.e., the initial configuration is exactly recovered after one spike emission). For long inhibitory pulses (small inhibitory decay rate $\beta$ ), we observed also stable period- 2 and higher order periodic solutions. Fig. 2 shows the transition from stable period- 1 to period- 2 solution in the $\alpha, \beta$ plane (from top to bottom). Higher order periodic solutions appear underneath that curve in the shaded area and result in a synchronous bursting dynamics.

\section{Linear stability analysis}

\subsection{General theory}

In this section, we present the stability analysis of a synchronous state in the period-1 regime. At variance with Ref. [20], we do not construct the corresponding map, which means that the phase-space dimension is not reduced by a suitable Poincaré section and the presence of a neutral direction is preserved. Actually, this property can be even used to double check the correctness of the final result.

Let us start introducing a stroboscopic representation and focus on a weakly perturbed synchronous configuration

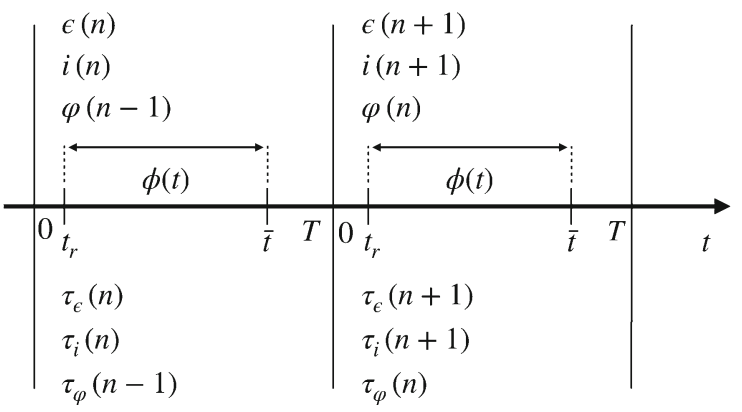

Fig. 3 An illustration of the perturbation analysis in time $t$ for the synchronous state

$E^{j}(n)=E_{r}+\epsilon^{j}(n)$

$I^{j}(n)=I_{r}+i^{j}(n)$

$\Phi^{j}(n)=\Phi_{r}+\varphi^{j}(n)$

where all variables are determined at the end of consecutive refractory periods. However, as shown in Fig. 3 and clarified in the following, it is convenient to refer $\varphi^{j}(n)$ to one period later with respect to $\epsilon^{j}(n)$ and $i^{j}(n)$. The fields $E_{r}=E_{\circ} \mathrm{e}^{-\alpha t_{r}}, I_{r}=I_{\circ} \mathrm{e}^{-\beta t_{r}}$, and the $\Phi_{r}=0$ do not depend on $n$, as the reference trajectory is periodic of period $T$. The overall perturbation can be represented as a $3 N$ dimensional vector $[\boldsymbol{\epsilon}(n), \boldsymbol{i}(n), \boldsymbol{\varphi}(n)]$. For the future sake of simplicity, it is convenient to introduce also a second representation $\boldsymbol{v}(n)=\left[\boldsymbol{\tau}_{\epsilon}(n), \boldsymbol{\tau}_{i}(n), \boldsymbol{\tau}_{\varphi}(n)\right]$, where

$\boldsymbol{\tau}_{\epsilon}(n)=\boldsymbol{\epsilon}(n) / \dot{E}_{r}$

$\boldsymbol{\tau}_{i}(n)=\boldsymbol{i}(n) / \dot{I}_{r}$,

$\boldsymbol{\tau}_{\varphi}(n)=\boldsymbol{\varphi}(n) / \dot{\Phi}_{r}$

and $\dot{E}_{r}, \dot{I}_{r}$ and $\dot{\Phi}_{r}$ all denote time derivates at the end of a refractory period. In practice, $\boldsymbol{\tau}_{x}$ corresponds to the time shift of the original trajectory to match the current perturbed state. The recursive transformation can be formally written as

$\boldsymbol{v}(n+1)=\mathbb{L} \boldsymbol{v}(n)$.

Our next task is to determine the operator $\mathbb{L}$. We start from the evolution equation of the excitatory field,

$E^{j}(n+1)=\mathrm{e}^{-\alpha T} E^{j}(n)+\alpha \sum_{k} G_{j, k} P_{k, k} \mathrm{e}^{-\alpha t^{k}(n)}$,

where $t^{k}(n)$ is the time elapsed since the arrival of the spike sent by the $k$ th neuron in the $n$th iterate.

Since the trajectory is close to the synchronous periodic orbit, $E^{j}(n+1)=E_{r}+\epsilon^{j}(n+1)$, and 
$t^{k}(n)=t_{r}+\tau_{\varphi}^{k}(n)$. Up to first order in the perturbations, Eq. (7) yields,

$$
\begin{aligned}
& \epsilon^{j}(n+1)=\mathrm{e}^{-\alpha T} \epsilon^{j}(n) \\
& -\alpha^{2} \mathrm{e}^{-\alpha t_{r}} \sum_{k} G_{j, k} P_{k, k} \tau_{\varphi}^{k}(n),
\end{aligned}
$$

or, in vector notations,

$\boldsymbol{\epsilon}(n+1)=A_{e} \boldsymbol{\epsilon}(n)-C_{e} \mathbf{G P} \boldsymbol{\tau}_{\varphi}(n)$

where

$A_{e}=\mathrm{e}^{-\alpha T} \quad, \quad C_{e}=\alpha^{2} \mathrm{e}^{-\alpha t_{r}}$.

A similar analysis of the inhibitory field leads to

$\boldsymbol{i}(n+1)=A_{i} \boldsymbol{i}(n)-C_{i} \mathbf{G}(\mathbb{1}-\mathbf{P}) \boldsymbol{\tau}_{\varphi}(n)$

where $\mathbb{1}$ is the $N \times N$ identity matrix, while

$$
A_{i}=\mathrm{e}^{-\beta T} \quad, \quad C_{i}=g \beta^{2} \mathrm{e}^{-\beta t_{r}} .
$$

Notice that, at variance with the previous case, there is an extra factor $g$ in the definition of $C_{i}$ to account for the larger amplitude of the inhibitory spikes.

Finally, we deal with phase dynamics. The core of the transformation is the mapping between the amplitude $\varphi(n)$ of the perturbation at time $t_{r}$ and the amplitude $\bar{\varphi}(n+1)$ at time $\bar{t}$, which can be formally written as

$\overline{\boldsymbol{\varphi}}(n+1)=S_{e} \boldsymbol{\epsilon}(n+1)+S_{i} \boldsymbol{i}(n+1)+S_{\phi} \boldsymbol{\varphi}(n)$.

This transformation is diagonal (it is the same for all components); the three unknown parameters, $S_{e}, S_{i}$, and $S_{\phi}$, can be determined by integrating the equation obtained from the linearization of Eq. (1). To separate the notation of the stroboscopic phase perturbation $\varphi(n)$ from the continuously developing phase perturbation between $t_{r}$ and $\bar{t}$, we introduce $\phi(t)$ for the later,

$$
\begin{aligned}
\dot{\phi} & =J \Gamma^{\prime}(\Phi)(E(t)-I(t)) \phi \\
& +J \Gamma(\Phi)\left[\mathrm{e}^{-\alpha\left(t-t_{r}\right)} \epsilon-\mathrm{e}^{-\beta\left(t-t_{r}\right)} i\right] .
\end{aligned}
$$

More precisely, upon setting $\left[\epsilon, i, \phi\left(t_{r}\right)\right]=[1,0,0]$, $[0,1,0]$, and $[0,0,1], \phi(\bar{t})$ corresponds to $S_{e}, S_{i}$, and $S_{\phi}$, respectively. Once $\bar{\varphi}(n+1)$ is known from Eq. (11), it can be transformed into the corresponding time shift

$\tau_{\varphi}(n+1)=\frac{\bar{\varphi}(n+1)}{\dot{\Phi}}$

where

$\dot{\bar{\Phi}}=1+J \Gamma(\bar{\Phi})\left(E_{\circ} \mathrm{e}^{-\alpha \bar{t}}-I_{\circ} \mathrm{e}^{-\beta \bar{t}}\right)$ is the time derivative of the phase in the point where the neuron stops feeling the action of the field. In between $\bar{t}$ and $T$, the oscillators evolve with the same velocity and no adjustment to the time shift can be expected. The transformation is completed by Eq. (5) which allows mapping $\varphi(n)$ into the corresponding time shift and obtaining

$$
\begin{aligned}
& \dot{\bar{\Phi}} \boldsymbol{\tau}_{\varphi}(n+1) \\
& =S_{e} \boldsymbol{\epsilon}(n+1)+S_{i} \boldsymbol{i}(n+1)+S_{\phi} \dot{\Phi}_{r} \boldsymbol{\tau}_{\varphi}(n) .
\end{aligned}
$$

With the help of Eqs. $(9,10)$, we obtain

$$
\begin{aligned}
& \dot{\bar{\Phi}} \boldsymbol{\tau}_{\varphi}(n+1)=A_{e} S_{e} \boldsymbol{\epsilon}(n)+A_{i} S_{i} \boldsymbol{i}(n) \\
& -\left[C_{e} S_{e} \mathbf{G P}+C_{i} S_{i} \mathbf{G}(\mathbb{1}-\mathbf{P})-S_{\phi} \dot{\Phi}_{r}\right] \boldsymbol{\tau}_{\varphi}(n) .
\end{aligned}
$$

or, in a more compact form,

$\dot{\bar{\Phi}} \boldsymbol{\tau}_{\varphi}(n+1)=A_{e} S_{e} \boldsymbol{\epsilon}(n)+A_{i} S_{i} \boldsymbol{i}(n)-\boldsymbol{M} \boldsymbol{\tau}_{\varphi}(n)$.

where $\mathbf{M}$ is an $N \times N$ matrix whose entries are defined as follows,

$\mathbf{M}_{j k}= \begin{cases}C_{e} S_{e}, & \text { if } k \rightarrow j, k \leq N_{E} \\ C_{i} S_{i}, & \text { if } k \rightarrow j, N_{E}<k \leq N \\ -S_{\phi} \dot{\Phi}_{r}, & \text { if } j=k \\ 0, & \text { no connection from } k \text { to } j \neq k\end{cases}$

For homogeneity reasons, it is convenient to express all of the three recursive relations in terms of the components of the $v$ vector,

$$
\begin{aligned}
\boldsymbol{\tau}_{\epsilon}(n+1)= & A_{e} \boldsymbol{\tau}_{\epsilon}(n)-\frac{C_{e}}{\dot{E}_{r}} \mathbf{G P} \boldsymbol{\tau}_{\varphi}(n) \\
\boldsymbol{\tau}_{i}(n+1)= & A_{i} \boldsymbol{\tau}_{i}(n)-\frac{C_{i}}{\dot{I}_{r}} \mathbf{G}(\mathbb{1}-\mathbf{P}) \boldsymbol{\tau}_{\varphi}(n) \\
\boldsymbol{\tau}_{\varphi}(n+1)= & A_{e} S_{e} \frac{\dot{E}_{r}}{\dot{\Phi}} \boldsymbol{\tau}_{\epsilon}(n)+A_{i} S_{i} \frac{\dot{I}_{r}}{\dot{\Phi}} \boldsymbol{\tau}_{i}(n) \\
& -\frac{\boldsymbol{M} \boldsymbol{\tau}_{\varphi}(n)}{\dot{\Phi}}
\end{aligned}
$$

Now, let us consider a homogeneous perturbation, such that $\boldsymbol{\tau}_{\epsilon}=\boldsymbol{\tau}_{i}=\boldsymbol{\tau}_{\varphi}$. This perturbation must be mapped exactly onto itself, since it corresponds to a homogenous time shift of the orbit. Let us see what this amounts to. From the first of the above equations, we have that

$1=A_{e}-C_{e} K_{e} / \dot{E}_{r}$ 
By looking at the definition of the various quantities, we can see that the equality is indeed satisfied. This is because $C_{e} / \dot{E}_{r}=-\left(1-A_{e}\right) / K_{e}$. Analogously, we can verify that $C_{i} / \dot{I}_{r}=-\left(1-A_{i}\right) / K_{i}$, so that we can rewrite the transformation as

$$
\begin{aligned}
\boldsymbol{\tau}_{\epsilon}(n+1)= & A_{e} \boldsymbol{\tau}_{\epsilon}(n)+\mathbf{0} \boldsymbol{\tau}_{i}(n)+\frac{1-A_{e}}{K_{e}} \mathbf{G P} \boldsymbol{\tau}_{\varphi}(n) \\
\boldsymbol{\tau}_{i}(n+1)= & \mathbf{0} \boldsymbol{\tau}_{\epsilon}(n)+A_{i} \boldsymbol{\tau}_{i}(n) \\
& +\frac{1-A_{i}}{K_{i}} \mathbf{G}(\mathbb{1}-\mathbf{P}) \boldsymbol{\tau}_{\varphi}(n) \\
\boldsymbol{\tau}_{\varphi}(n+1)= & B_{e} \boldsymbol{\tau}_{\epsilon}(n)+B_{i} \boldsymbol{\tau}_{i}(n)-\frac{\boldsymbol{M} \boldsymbol{\tau}_{\varphi}(n)}{\dot{\Phi}}
\end{aligned}
$$

where $B_{e}=A_{e} S_{e} \dot{E}_{r} / \dot{\bar{\Phi}}$ and $B_{i}=A_{i} S_{i} \dot{I}_{r} / \dot{\bar{\Phi}}$.

By playing the same game of homogenous perturbations with the last equation of Eq. (16), we find that $\dot{\bar{\Phi}}=\dot{E}_{r} S_{e}+\dot{I}_{r} S_{i}+\dot{\Phi}_{r} S_{\phi}$.

Direct numerical simulations confirm that this condition is satisfied, as it should, since it implies that a homogeneous shift of the phase of all oscillators is time invariant.

Altogether Eq. (17) is a representation of the linear operator $\mathbb{L}$ formally introduced in Eq. (6). The eigenvalues of $\mathbb{L}$ are the so-called Floquet multipliers $Z_{i}$; the synchronous solution is stable if the modulus of all multipliers is smaller than $1 .^{2}$ One can equivalently refer to the Floquet exponents $\lambda_{i}=\log \left|Z_{i}\right|$ that we also call Lyapunov exponents with a slight stretch of the notations.

For $\alpha, \beta \gg 1$, the fields are exponentially small when the neurons reach the threshold. In this limit, the fields behave as slaved variables and their contribution can be neglected in the stability analysis, which reduces to diagonalizing an $N \times N$ matrix,

$\boldsymbol{\tau}_{\varphi}(n+1)=-\boldsymbol{M} \boldsymbol{\tau}_{\varphi}(n)$,

(notice that $\dot{\bar{\Phi}}$ can be safely set equal to 1 , as the coupling is indeed negligible at time $\bar{t}$ ).

\subsection{Transversal Lyapunov exponent}

A simpler approach to assess the stability of the synchronous regime consists in investigating the stability

${ }^{2}$ With the exception of the unit multiplier associated with a time shift of the trajectory. of a single neuron subject to the external periodic modulation resulting from the network activity. The corresponding growth rate $\lambda_{c}$ of infinitesimal perturbations is called transversal or conditional Lyapunov exponent. In mean-field models, this approach leads to the same result obtained by implementing a more rigorous theory which takes into account mutual coupling. Let the time shift at the end of a refractory period be equal to $\tau_{r}$; the corresponding phase shift is therefore

$\phi\left(t_{r}\right)=\dot{\Phi}\left(t_{r}\right) \tau_{r}=\left\{1+J \Gamma(0)\left[E\left(t_{r}\right)-I\left(t_{r}\right)\right]\right\} \tau_{r}$.

From time $t_{r}$ up to time $\bar{t}$, the phase shift evolves according to the simplified version of Eq. (12),

$\dot{\phi}=J \Gamma^{\prime}(\Phi)(E(t)-I(t)) \phi$,

where we have neglected the variation of field dynamics, since the field is treated as an external forcing. As a result,

$\phi(\bar{t})=\mathrm{e}^{D} \phi\left(t_{r}\right)$,

where, with reference to the PRC Eq. (3),

$D=\frac{J I_{\circ}}{\beta}\left[\mathrm{e}^{-\beta \bar{t}}-\mathrm{e}^{-\beta t_{r}}\right]-\frac{J E_{\circ}}{\alpha}\left[\mathrm{e}^{-\alpha \bar{t}}-\mathrm{e}^{-\alpha t_{r}}\right]$

The corresponding time shift is

$\bar{\tau}=\frac{\phi(\bar{t})}{\dot{\Phi}}$

The shift $\bar{\tau}$ carries over unchanged until first the threshold $\phi=1$ is crossed, and then, the new refractory period ends. Accordingly, from Eqs. $(19,21)$, the expansion $R$ of the time shift over one period (a sort of Floquet multiplier) can be written as

$R=\frac{\bar{\tau}}{\tau_{r}}=\frac{1+J \Gamma(0)\left[E\left(t_{r}\right)-I\left(t_{r}\right)\right]}{\dot{\Phi}} \mathrm{e}^{D}$

This formula is substantially equivalent to Eq. (54) of Ref. [20] $\left(\Lambda_{i i}\right.$ corresponds to $R$ ), obtained while studying a single population under the action of $\alpha$-pulses. An additional marginal difference is that while in Ref. [20] the single neuron dynamics is described by a nonuniform velocity field $F(x)$ and homogeneous coupling strength, here we refer to a constant velocity and a phase-dependent PRC, $\Gamma(\phi)$. is

The corresponding conditional Lyapunov exponent 


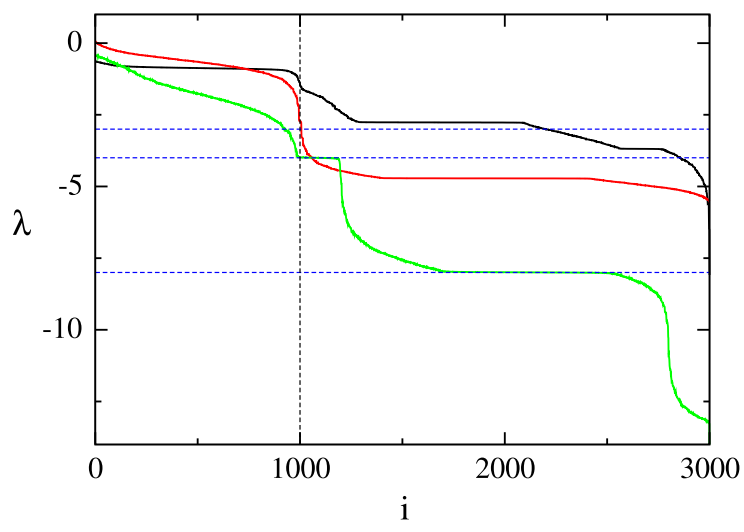

Fig. 4 The total Lyapunov spectrum, for $(\alpha, \beta)$ equal to $(4,3)$, $(4,4)$, and $(4,8)$ - top to bottom; the coupling strength is $J=$ 0.03 , as for all of our simulations, while $N=1000$. The three horizontal dashed lines correspond to the three different rates used to identify $\alpha$ and $\beta$ values. The vertical dashed line separates the part of the spectrum which, for large $\alpha$ and $\beta$ values are expected to be related to the actual network structure

$$
\begin{aligned}
\lambda_{c} & =\frac{\ln |R|}{T} \\
& =\frac{D+\ln \left|\left[1+J \Gamma(0)\left(E\left(t_{r}\right)-I\left(t_{r}\right)\right)\right] / \dot{\bar{\Phi}}\right|}{T} .
\end{aligned}
$$

It is the sum of two contributions: the former one accounting for the linear stability of the phase evolution from reset to threshold $(D / T)$; the latter term arises from the different velocity (frequency) exhibited at threshold and at the end of the refractory period. Notice that in the limit of short pulses, the field amplitude at time $\bar{t}$ can be set equal to zero, thereby neglecting the corresponding exponential terms in Eq. (22) and assuming $\dot{\Phi}=0$.

\section{Application}

We now implement the general formalism in the case of the PRC defined by Eq. (3), considering a network with $N=1000$ neurons, a $10 \%$ connectivity (i.e., $K=100$ with $K_{e}=80$ and $K_{i}=20$ ), and $g=5$; the coupling strength is assumed to be $J=0.03$, while the refractory time is $t_{r}=0.03$. This setup, characterized by a slight prevalence of inhibition $\left(g K_{i} \gtrsim K_{e}\right)$, is often adopted in the study of balanced regimes (see, e.g., [23]).

The resulting Floquet spectra are presented in Fig. 4 for three different pairs of not-too-large $\alpha$ and $\beta$ values. Rather than diagonalizing the matrix defined by Eq. (17), the 3,000 exponents have been determined

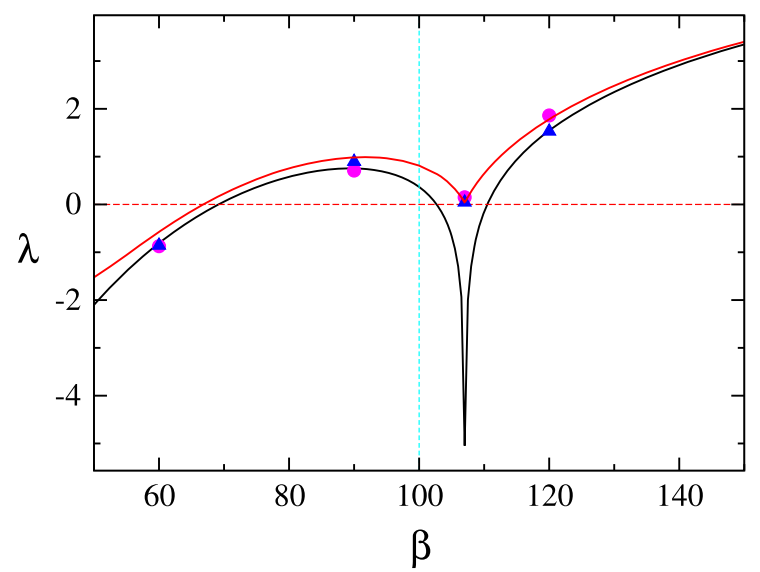

Fig. 5 The maximal Lyapunov/Floquet exponent $\lambda_{M}$ (upper red curve) vs. $\beta$ for a network of $N=10,000$, with $\alpha=100$ and the other parameters set as in the previous figure. The black curve corresponds to the transversal/conditional exponent $\lambda_{c}$, while full dots and triangles result from the computation of the finiteamplitude Lyapunov exponent $\lambda_{f}$ for $\delta_{p}=10^{-2}$ and $10^{-3}$, respectively

by implementing a standard algorithm for the computation of Lyapunov exponents [27]. The larger are $\alpha$ and $\beta$, the more step-like is the spectral shape, the two lower steps being located around the decay rate (i.e., the inverse pulse-width) of the pulses (see the three horizontal dashed lines, which correspond to $\lambda=-3$, -4 , and -8 , respectively). This is sort of expected, since the field dynamics basically amounts to a relaxation process controlled by the respective decay rate. Anyhow, since the overall stability is determined by the largest exponents, it is sufficient to restrict the analysis to the first part of the spectrum (to the left of the vertical dashed line in Fig. 4), which, in the limit of large $\alpha$ and $\beta$, can be directly determined by diagonalizing the matrix defined in Eq. (18).

The dependence of the maximum exponent $\lambda_{M}$ on the (inverse) pulse-width of the inhibitory spikes is reported in Fig. 5 (see the upper red curve). In this case, the Floquet exponent has been obtained by diagonalizing the matrix in Eq. (18) for a system size $N=10,000$ and a connectivity $K=1000\left(K_{e}=800, K_{i}=200\right)$.

The vertical dashed line corresponds to the symmetric case, where both excitatory and inhibitory neurons have the same width (and shape). Interestingly, the stability, determined by the largest nonzero exponent, (the always present $\lambda=0$, corresponds to the neutral stability associated with a time shift of the trajectory) depends strongly on the relative excitatory/inhibitory 
pulse width and can even change sign: the synchronous solution is stable below $\beta=67^{3}$. Additionally, there is evidence of a sort of singularity around $\beta=107$, when the inhibitory spikes are slightly shorter than the excitatory ones.

Given the finite dimension of the matrices, sampleto-sample fluctuations are expected. Such fluctuations are, however, rather small, as testified by the smoothness of the red curve in Fig. 5. In fact, the single values of the Floquet exponents have been obtained not only by varying the (inhibitory) pulse-width, but also considering different network realizations. Although small, the fluctuations prevent drawing definite conclusions about the singularity seemingly displayed by the derivative of $\lambda_{M}(\beta)$ around $\beta=107$.

In the limit of a fully connected network, we expect a perfectly degenerate spectrum (all directions are mutually equivalent) and $\lambda_{M}$ equal to the conditional Lyapunov exponent $\lambda_{c}$ defined in Eq. (24). The lower black curve reported in Fig. 5 corresponds to $\lambda_{c}$; except for a narrow region around $\beta=107, \lambda_{c}$ is always close to (lower than) $\lambda_{M}$. This means that the mean-field approximation still works pretty well in a network of 10,000 neurons with a $10 \%$ connectivity.

The explicit formula Eq. (24) helps also to shed light on the $\beta$ dependence of the network stability. The main responsible for the qualitative changes observed around $\beta=107$ is the logarithmic term, arising from the difference between the velocity at threshold (equal to 1 , irrespective of the $\beta$-value) and the velocity at the end of the refractory period. This latter velocity is determined by the effective field $E_{\text {eff }}\left(t_{r}\right)=E\left(t_{r}\right)-I\left(t_{r}\right)$ which in turn strongly depends on the relative pulse-width. The time dependence of $E_{\text {eff }}$ can be appreciated in Fig. 6, where we report the trace for three different $\beta$ values $(60,90$, and 120) and the same $\alpha=100$. There, we see that even the sign of the effective field may change; for $\beta=120, E_{\text {eff }}$ is initially negative because inhibition dominates, but above $t=0.02<t_{r}$ the slower decay of the excitatory pulses takes over, so that the effective field amplitude is positive at the end of refractoriness. For $\beta=90<\alpha=100$, inhibition prevails at all times and the effective field is thereby negative for $t=t_{r}$. Finally, for $\beta=60$, excitation initially prevails, but inhibition takes soon over.

\footnotetext{
${ }^{3}$ For the sake of completeness, notice that by further decreasing $\beta$ the stability changes again.
}

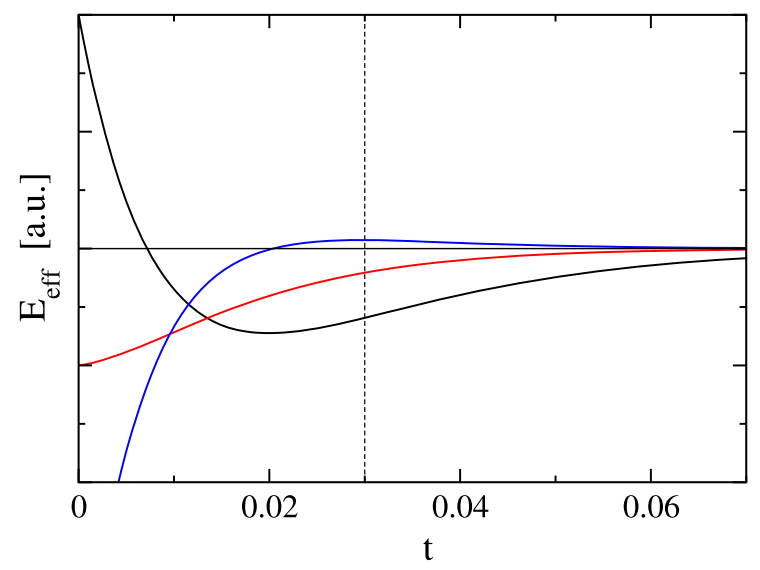

Fig. 6 Effective field shape for $\alpha=100$ and $\beta=60$ (black), $\beta=90$ (red), and $\beta=120$ (blue). The vertical dashed line identifies the end of the refractory period

From Eq. (24), we see that the sign of the logarithmic contribution changes depending whether the argument is smaller or larger than 1. More precisely, if the effective field is negative but larger than $-2 /(J \Gamma(0))$, the discontinuity of the velocity tends to stabilize the synchronous regime; if $E_{\text {eff }}\left(t_{R}\right)=-1 / J \Gamma(0)$, the orbit is even superstable, i.e., the Lyapunov exponent is infinitely negative. This is precisely what happens for $\beta \approx 107$. Altogether, the $\beta$ interval around 107 separates the region where the expansion/contraction factor is positive (to the right), from the region where it is negative (to the left).

The sign of the multiplier has a simple explanation: $1+J \Gamma(0) E_{\mathrm{eff}}\left(t_{r}\right)<0$ means that the phase velocity is negative at the end of the refractory period. Therefore, if one follows two nearby neurons-one leading over the other before reaching the threshold - then at the end of refractoriness, the leading neuron becomes the lagging one, as they initially move in the "wrong" direction" This explains how the pulse-width may affect the stability.

So far we have referred to the Floquet exponents, without paying attention to the phase of the multipliers. In Fig. 7, we report both real and imaginary part of all eigenvalues for four different $\beta$ values.

For $\beta=60$ and 90, the eigenvalues (except for $Z=(1,0))$ are distributed within a circle (see panels a and b). This is reminiscent of Girko's theorem [28], which states that the eigenvalues of an $N \times N$

\footnotetext{
${ }^{4}$ Later, the velocity changes sign becoming positive, but this does not modify the ordering.
} 


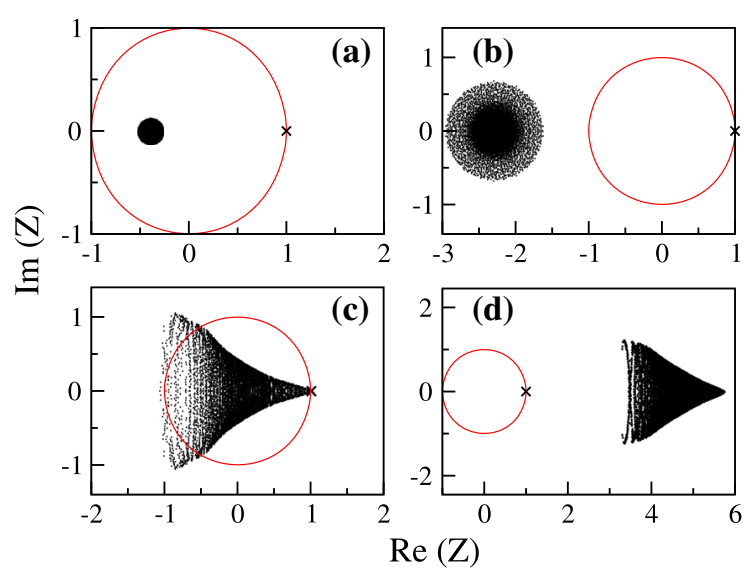

Fig. 7 The distribution of the complex eigenvalues (black dots) for short pulses, $N=10,000, \alpha=100$, and $J=0.03$. The red circle highlights the unit circle. The black cross at $(1,0)$ singles out the always present eigenvalue associated with the periodic motion. The four panels represent the results for different decay rates of the inhibitory pulses $\beta$. In particular $\beta=60,90,107$ and 120 for panel (a) - (d), respectively

random matrix with independent and identically distributed entries (with zero mean zero and variance equal to $1 / N$ ) are uniformly distributed over the unit disc. However, it is not obvious how to adapt/extend this theorem to the present context, since the matrix $\mathbf{M}$ although being random does not satisfy several of the required assumptions, starting from the off-diagonal elements which take only three different values and their average is nonzero.

Returning to Fig. 7, we see that for $\beta=60$ all the eigenvalues lie within the unit circle, meaning that the synchronous solution is stable, while for $\beta=90$ all eigenvalues lie outside, meaning it is fully unstable: any perturbation is amplified!

Above $\beta=100$, the spectrum changes shape, becoming funnel-like: for $\beta=120$ (panel d), all eigenvalues sit again outside the unit circle, meaning that the synchronous solution is fully unstable. Interestingly, for $\beta=107$ (panel $\mathrm{c}$ ), the funnel is almost entirely contained within the unit circle, so that the resulting (weak) instability is due to few complex eigenvalues detected on the upper-left and lower-left corners of the funnel. As an additional remark, we can see that the eigenvalue with largest modulus (i.e., the one determining the stability) is real and negative for $\beta=60$ and 90 , while it is real and positive for $\beta=120^{5}$. This

\footnotetext{
${ }^{5}$ In practice, depending on the network realization, the leading eigenvalues may have a small imaginary component.
}

is coherent with the behavior of the sign of the multiplier $R$ (see Eq. (23)), which changes from positive to negative, while decreasing $\beta$. The qualitative differences observed in the region around $\beta=107$ suggest that the "singular" behavior exhibited by $\lambda_{M}$ is the signature of a true transition associated with a change of the spectral structure.

Finally, a few words about the leading eigenvector. It must possess some special features which are responsible for its larger expansion rate. However, we have not found any correlation with obvious indicators such as an anomalously large outgoing connectivity. We have only observed that the vector components are distributed in a Gaussian way with zero average.

\subsection{Finite-amplitude perturbations}

Finally, we have directly investigated the stability of the synchronous regime, by studying the evolution of small but finite perturbations under the action of the model Eqs. (1-2) in the limit of short pulses. By following the same strategy developed in tangent space, the perturbation amplitude has been quantified as the temporal shift at a specific moment. We find it convenient to identify the specific moment with the threshold passing time $t^{L}(n)$ of the last neuron (in the $n$th period). Provided the perturbation is small enough, all neurons are still in the refractory period and their phase is equal to 0 when the time is taken. The temporal shift of the $j$ th neuron can be defined as $\delta_{j}=t^{L}(n)-t_{j}(n)$, where $t_{j}(n)$ is its $n$th passing time. The perturbation amplitude can be finally defined as the standard deviation $\delta_{p}(n)$ of all temporal shifts. Given an initial distribution with a fixed $\delta_{p}(0)$, it is let to evolve to determine its value once the new set of spiking times is over. The ratio $R_{f}=\delta_{p}(1) / \delta_{p}(0)$ represents the contraction or expansion factor over one period $T$. Afterward, the standard deviation is rescaled to the original value $\delta_{p}(0)$ to avoid it becoming either too large to be affected by nonlinear effects or too small to be undetectable. We have found that $\delta_{p}(0)=10^{-3}$, or $10^{-2}$ suffices to ensure meaningful results. The corresponding (finite amplitude) Lyapunov exponent $\lambda_{f}$ is finally obtained by iterating this procedure to let the perturbation converge along the most expanding direction and thereby computing $\lambda_{f}=\ln \left|R_{f}\right| / T$. We have found that 50 iterates suffice to let the transient die out.

A crucial point is the integration time step if the model is evolved by implementing an Euler algorithm. 
In fact, the time step must be much smaller than the separation between spike-times, since they have to be well resolved. We have set the Euler integration time step $\Delta t$ at least 100 times smaller than the standard deviation of the perturbation $\delta_{p}(0)$. The numerical results are plotted in Fig. 5 for four different $\beta$ values (see the symbols): they confirm the theoretical predictions.

\section{Conclusions and open problems}

In this paper, we have developed a formalism to assess the stability of synchronous regimes in sparse networks of two populations of oscillators coupled via finitewidth pulses. The problem is reduced to the determination of the spectral properties of a suitable class of sparse random matrices. Interestingly, we find that the relative width of excitatory and inhibitory spikes plays a crucial role even in the limit of narrow spikes, up to the point that the stability may qualitatively change. This confirms once more that the $\delta$-spike limit is singular and it is necessary to include the spike width into the modeling of realistic neuronal networks.

Our analytical treatment has allowed constructing the stability matrix, but the derivation of an analytical solution of the spectral problem remains an open problem. The conditional Lyapunov exponent provides an approximate expression for the maximum Floquet exponent. It is quite accurate in a broad range of pulsewidths but fails to predict the weak instability occurring when inhibitory pulses are slightly narrower than excitatory ones. For relatively wider inhibitory pulses, numerical simulations suggest that it will be worth exploring the possibility to extend the circular law of random matrices to sparse matrices of the type derived herewith.

While mean-field models are characterized by a degenerate spectrum (all directions are equally stable), here the degeneracy is lifted by the randomness associated with the sparse connectivity. It is therefore desirable to understand which features make some directions so special as to be characterized by a minimal stability. This is probably related to the presence of closed loops of connections among oscillators which sustain an anticipated or retarded firing activity. Further studies are required.

Acknowledgements Afifurrahman was supported by the Ministry of Finance of the Republic of Indonesia through the Indone- sia Endowment Fund for Education (LPDP) (grant number: PRJ2823/LPDP/2015).

\section{Compliance with ethical standards}

Conflict of interest The authors declare that they have no conflict of interest.

Open Access This article is licensed under a Creative Commons Attribution 4.0 International License, which permits use, sharing, adaptation, distribution and reproduction in any medium or format, as long as you give appropriate credit to the original author(s) and the source, provide a link to the Creative Commons licence, and indicate if changes were made. The images or other third party material in this article are included in the article's Creative Commons licence, unless indicated otherwise in a credit line to the material. If material is not included in the article's Creative Commons licence and your intended use is not permitted by statutory regulation or exceeds the permitted use, you will need to obtain permission directly from the copyright holder. To view a copy of this licence, visit http://creativecommons.org/licenses/ by/4.0/.

\section{References}

1. Blekhman, I.I., Landa, P.S., Rosenblum, M.G.: Synchronization and chaotization in interacting dynamical systems. Appl. Mech. Rev. 48(11), 733 (1995). https://doi.org/10. 1115/1.3005090

2. Blekhman, I., Fradkov, A., Nijmeijer, H., Pogromsky, A.: On self-synchronization and controlled synchronization. Syst. Control Lett. 31(5), 299 (1997). https://doi.org/10.1016/ S0167-6911(97)00047-9

3. Filatrella, G., Nielsen, A.H., Pedersen, N.F.: Analysis of a power grid using a Kuramoto-like model. Eur. Phys. J. B 61(4), 485 (2008). https://doi.org/10.1140/epjb/ e2008-00098-8

4. Hadley, P., Beasley, M.R.: Analysis of a power grid using a Kuramoto-like model. Appl. Phys. Lett. 50(10), 621 (1987). https://doi.org/10.1063/1.98100

5. Javaloyes, J., Perrin, M., Politi, A.: Collective atomic recoil laser as a synchronization transition. Phys. Rev. E 78, 011108 (2008). https://doi.org/10.1103/PhysRevE.78. 011108

6. Izhikevich, E.M.: The Dynamical Systems in Neuroscience: Geometry of Excitability and Bursting. Computational Neuroscience Series. MIT Press, Cambridge (2006)

7. Pikovsky, A., Rosenblum, M., Kurths, J.: Synchronization: A Universal Concept in Nonlinear Sciences. Cambridge Nonlinear Science Series. Cambridge University Press, Cambridge (2001). https://doi.org/10.1017/ CBO9780511755743

8. Winfree, A.T.: The Geometry of Biological Time. Interdisciplinary Applied Mathematics, vol. 12, 2nd edn. Springer, New York (2001)

9. van Vreeswijk, C.: Partial synchronization in populations of pulse-coupled oscillators. Phys. Rev. E 54, 5522 (1996). https://doi.org/10.1103/PhysRevE.54.5522 
10. Panaggio, M.J., Abrams, D.M.: Chimera states: coexistence of coherence and incoherence in networks of coupled oscillators. Nonlinearity 28(3), R67 (2015)

11. Golomb, D., Hansel, D., Shraiman, B., Sompolinsky, H.: Clustering in globally coupled phase oscillators. Phys. Rev. A 45, 3516 (1992). https://doi.org/10.1103/PhysRevA.45. 3516

12. Abbott, L.F., van Vreeswijk, C.: Asynchronous states in networks of pulse-coupled oscillators. Phys. Rev. E 48, 1483 (1993). https://doi.org/10.1103/PhysRevE.48.1483

13. Golomb, D., Hansel, D., G, M.: in Neuro-informatics and Neural Modeling, ed. by F. Moss, S. Gielen. Elsevier, Amsterdam, p. 887-968 (2001)

14. Politi, A., Rosenblum, M.: Equivalence of phase-oscillator and integrate-and-fire models. Phys. Rev. E 91, 042916 (2015). https://doi.org/10.1103/PhysRevE.91.042916

15. Mirollo, R.E., Strogatz, S.H.: Synchronization of pulsecoupled biological oscillators. SIAM J. Appl. Math. 50(6), 1645 (1990). https://doi.org/10.1137/0150098

16. Van Vreeswijk, L.F., Abbott, C., Ermentrout, G.B.: When inhibition not excitation synchronizes neural firing. J. Comput. Neurosci. 1, 313 (1994). https://doi.org/10.1007/ BF00961879

17. Hansel, D., Mato, G., Meunier, C.: Synchrony in excitatory neural networks. Neural Comput. 7(2), 307 (1995). https:// doi.org/10.1162/neco.1995.7.2.307

18. Goel, P., Ermentrout, B.: Synchrony, stability, and firing patterns in pulse-coupled oscillators. Physica D Nonlinear Phenomena 163(3), 191 (2002). https://doi.org/10.1016/ S0167-2789(01)00374-8

19. Zillmer, R., Livi, R., Politi, A., Torcini, A.: Stability of the splay state in pulse-coupled networks. Phys. Rev. E 76, 046102 (2007). https://doi.org/10.1103/PhysRevE.76. 046102

20. Olmi, S., Politi, A., Torcini, A.: Linear stability in networks of pulse-coupled neurons. Front. Comput. Neurosci. 8, 8 (2014). https://doi.org/10.3389/fncom.2014.00008
21. Timme, M., Wolf, F.: The simplest problem in the collective dynamics of neural networks: is synchrony stable? Nonlinearity 21(7), 1579 (2008). https://doi.org/10.1088/ 0951-7715/21/7/011

22. Brunel, N.: Dynamics of sparsely connected networks of excitatory and inhibitory spiking neurons. J. Comput. Neurosci. 8(3), 183 (2000). https://doi.org/10.1023/A: 1008925309027

23. Ostojic, S.: Two types of asynchronous activity in networks of excitatory and inhibitory spiking neurons. Nat. Neurosci. 17(4), 594 (2014). https://doi.org/10.1038/nn.3658

24. Ullner, E., Politi, A., Torcini, A.: Ubiquity of collective irregular dynamics in balanced networks of spiking neurons. Chaos Interdiscip. J. Nonlinear Sci. 28(8), 081106 (2018). https://doi.org/10.1063/1.5049902

25. Politi, A., Ullner, E., Torcini, A.: Collective irregular dynamics in balanced networks of leaky integrate-and-fire neurons. Eur. Phys. J. Spec. Top. 227(10), 1185 (2018). https://doi. org/10.1140/epjst/e2018-00079-7

26. Ullner, E., Politi, A.: Self-sustained irregular activity in an ensemble of neural oscillators. Phys. Rev. X 6, 011015 (2016). https://doi.org/10.1103/PhysRevX.6.011015

27. Pikovsky, A., Politi, A.: Lyapunov Exponents: A Tool to Explore Complex Dynamics. Cambridge University Press, Cambridge (2016). https://doi.org/10.1017/ CBO9781139343473

28. Girko, V.L.: Circular law. Theory Probab. Appl. 29(4), 694 (1984). https://doi.org/10.1137/1129095

Publisher's Note Springer Nature remains neutral with regard to jurisdictional claims in published maps and institutional affiliations. 